\title{
Fatores associados às internações hospitalares no Brasil
}

\author{
Analysis of hospital admissions associated \\ factors in Brazil
}

Mônica Silva Monteiro de Castro 1

Cláudia Travassos 2

Marília Sá Carvalho ${ }^{3}$

\footnotetext{
1 Escola Nacional de Saúde Pública, Fundação Oswaldo Cruz (ENSP/Fiocruz). Rua Leopoldo Bulhões, 1.480/810. 21041-210 Manguinhos Rio de Janeiro RJ. mmcastro@uai.com.br 2 Departamento de Informações em Saúde, Centro de Informação Científica e Tecnológica, Fiocruz.

3 Departamento de Epidemiologia e Métodos Quantitativos em Saúde, ENSP/Fiocruz.
}

Abstract The objective was to identify hospital admission associated factors in Brazil, analyzing if that utilization is equitable and identifying which characteristics are associated with heavy users. We analyzed data from the 1998 National Household Survey, using logistic regression and multinomial logistic regression, with normalized weights and statistical techniques to correct for design effect. We used Andersen's Behavioral Model as the theoretical model for this analysis. In model adjusted for health needs and enabling factors, people with smaller income had more chance of being admitted to hospital; and in model adjusted only for health needs people with larger income had more chance. All need variables were less related to use for people with two hospital admissions, in comparison with those with more than two admissions; and there was no association between social variables and occurrence of two admissions, but this association occurred for three or more admissions. The reduction of social inequalities in the aspects that "enable" people to use hospital admissions would reduce inequalities in this use. A health system that offers a "regular health service", besides low or any payment in the moment of use, would have a positive impact in the equity of hospital admission utilization in Brazil.

Key words Hospital services utilization, Heavy users, Equity
Resumo O objetivo foi identificar fatores associados às admissões hospitalares no Brasil, analisando se essa utilização é eqüitativa e identificando características associadas aos grandes usuários. A PNAD/1998 foi analisada, utilizando regressão logística e regressão logística multinomial, com pesos normalizados e técnicas estatísticas para correção do efeito de desenho. O modelo teórico utilizado foi o Comportamental de Andersen. No modelo ajustado por necessidade de saúde e fatores capacitantes, pessoas com menor renda apresentaram maior chance de se internar; o contrário ocorreu no modelo ajustado somente por necessidade de saúde. Todas as variáveis de necessidade mostraram-se menos relacionadas ao uso nas pessoas com duas internações, em comparação com aquelas com mais do que duas internações. Não houve associação entre variáveis sociais e ocorrência de duas internações, mas essa associação ocorreu para três ou mais internações. A redução das desigualdades sociais nos aspectos que "capacitam" ao uso de admissões hospitalares reduziria as desigualdades neste uso. Um sistema de saúde que ofereça um "serviço de uso regular", além de baixo ou nenhum pagamento no ato do consumo, seriam medidas de impacto positivo na eqüidade do consumo de serviços hospitalares no Brasil.

Palavras-chave Utilização de serviços hospitalares, Grandes usuários, Eqüidade 


\section{Introdução}

O cuidado hospitalar é importante objeto de pesquisa em serviços de saúde, devido ao seu papel central na assistência e ao seu alto custo. $\mathrm{O}$ entendimento dos fatores associados à utilização de serviços hospitalares é fundamental para a discussão de políticas voltadas para o aumento da eqüidade do sistema de saúde brasileiro. Eqüidade é um conceito complexo, com diversas formas de operacionalização. As normas do Sistema Único de Saúde (SUS) estão de acordo com a definição de eqüidade como "tratamentos de saúde iguais para necessidades de saúde iguais" (Porto, 1997).

O Brasil tem um sistema de saúde misto, composto pelo SUS, com financiamento público, e pela denominada Medicina Suplementar - convênios, seguros e medicina privada. Por princípio, o SUS deve assistir à população brasileira de forma universal; na prática, sua cobertura varia de lugar para lugar no país e entre grupos sociais (Travassos, 1997). O setor da medicina suplementar opera segundo a lógica de mercado e é pautado por princípios claramente não eqüitativos.

Os serviços de saúde podem ser estudados tomando como base o Modelo Comportamental desenvolvido por Andersen (1968), com o objetivo de orientar a compreensão do uso, a estimativa da eqüidade no acesso e a definição de políticas de promoção nesse sentido. Neste modelo, o uso de serviços de saúde é função da predisposição dos indivíduos; de fatores capacitantes, que possibilitam ou impedem este uso e da necessidade de cuidados de saúde. São características predisponentes: fatores demográficos, aqueles relacionados à estrutura social e fatores culturais. Fatores capacitantes, individuais e da área devem estar presentes. Para que o uso de serviços de saúde ocorra é preciso que existam instalações, equipamentos e equipes de saúde, e que os indivíduos tenham os meios e os conhecimentos necessários para chegar aos serviços e fazer uso deles. A necessidade de serviços de saúde pode ser aquela indicada pelo próprio indivíduo, a chamada necessidade de saúde auto-avaliada, ou avaliada pelo profissional de saúde (Andersen, 1995).

A operacionalização do Modelo Comportamental inclui nos fatores predisponentes: sexo e idade (demográficos), educação, ocupação e raça (estrutura social); e nos fatores capacitantes, renda, cobertura por seguro de saúde, existência de fonte regular de cuidado, tempos de viagem até o serviço e de espera até o atendimento, entre outros. O uso de serviços é medido em unidades de consultas médicas de internações hospitalares, podendo ser especificada para um determinado problema de saúde ou tipo de serviço (Andersen, 1995).

$\mathrm{O}$ acesso potencial é definido pelos fatores capacitantes e a efetivação do acesso é o uso real dos serviços de saúde. A utilização seria eqüitativa quando a maior parte da variação da utilização fosse explicada pelos fatores demográficos e de necessidade, e não eqüitativa quando a maior parte dessa variação fosse explicada pela estrutura social, crenças de saúde e fatores capacitantes (Andersen, 1995).

Idade e sexo são características naturalmente associadas ao uso de serviços hospitalares, em razão da sua influência biológica na morbimortalidade (Hulka \& Wheat, 1985), e assim, as análises de utilização de serviços hospitalares devem ser ajustadas por idade e sexo. As pessoas de pior condição socioeconômica têm mais necessidades de saúde (Evans et al., 1994) e as pessoas que têm mais necessidades de saúde usam mais serviços de saúde (Hulka \& Wheat, 1985). Porém, um maior uso de serviços de saúde pelas pessoas de pior situação socioeconômica e de maior necessidade depende de funcionamento do sistema de saúde.

Nos Estados Unidos, a pobreza e o desemprego apresentaram associação positiva e a educação, associação negativa com as taxas de internação (McLaughlin et al., 1989; McMahon et al., 1993), e o efeito das características socioeconômicas nas taxas de hospitalização era maior nos adultos jovens e diminuía com a idade (Hofer et al., 1998). No Canadá, as pessoas de menor renda e com mais necessidades de saúde usaram mais serviços hospitalares (Newbold et al., 1995; Anderson, 1997; Glazier et al., 2000), mas maiores taxas de cirurgia foram encontradas em áreas de maior escolaridade e maior proporção de indivíduos de origem canadense, americana ou inglesa (Roos \& Roos, 1982). Na Espanha, não foram encontradas desigualdades nas internações hospitalares entre classes sociais, medidas por uma adaptação da Classificação de Classes Sociais, baseada nas ocupações, desenvolvida na Inglaterra (Borrell et al., 2000). No Rio de Janeiro, o fator explicativo mais importante da utilização de serviços de saúde por idosos foi a necessidade medida pela morbidade auto-referida, e o uso de serviços em áreas de pior condição econômica dependeu mais da renda individual do que em 
áreas de melhor condição econômica (Pinheiro \& Travassos, 1999).

Uma característica do uso de serviços de saúde, incluindo os serviços hospitalares, é que uma pequena proporção de indivíduos, denominados grandes usuários de serviços de saúde, é responsável por uma parte grande e desproporcional do uso. Outro aspecto importante é que os grandes usuários tendem a permanecer como grandes usuários ao longo do tempo (Densen et al., 1959; McFarland et al., 1985). Estudo de utilização de serviços hospitalares nos Estados Unidos indicou que os grandes usuários persistentes têm mais idade, referem pior estado de saúde, apresentam maior número de sintomas físicos e um grau maior de estresse psicológico, especialmente depressão (McFarland et al., 1985; Freeborn et al., 1990).

Os objetivos deste trabalho foram identificar os fatores associados às internações hospitalares no Brasil, verificando quais características explicam as variações na ocorrência de internação entre as pessoas e se essas variações caracterizam uma situação de iniqüidade na utilização de serviços hospitalares no país, além de identificar o que caracteriza o os grandes usuários de serviços hospitalares no Brasil.

\section{Metodologia}

Os dados analisados são provenientes da Pesquisa Nacional por Amostra de Domicílios de 1998 (PNAD/1998), inquérito populacional realizado anualmente pelo Instituto Brasileiro de Geografia e Estatística (IBGE). São excluídas da amostra as áreas rurais da região Norte do país (Rondônia, Acre, Amazonas, Roraima, Pará e Amapá). O período de referência de doze meses foi de outubro de 1997 a setembro de 1998 (IBGE, 2000). O Suplemento Saúde da PNAD/ 1998 abordou aspectos de morbidade, cobertura por planos de saúde, acesso a serviços de saúde, utilização de serviços de saúde, internações no sistema público e privado e gasto privado em saúde (Travassos et al., 2000a).

O banco de dados da PNAD/1998 contém informações sobre 344.975 indivíduos. Em 36,8\% das vezes o informante do suplemento saúde foi a própria pessoa, em $61,0 \%$ outra pessoa moradora do domicílio e em $2,2 \%$ outra pessoa não moradora do domicílio. Foram excluídos da análise $112(0,03 \%)$ indivíduos para os quais a informação sobre a ocorrência ou não de internação hospitalar no último ano estava ausente.
Na parte descritiva da análise foram estudadas todas as 23.900 internações informadas. Na descrição da última internação ocorrida no período de referência foram utilizadas as seguintes variáveis: motivo principal da internação (clínico, cirúrgico, partos e outros), tempo de permanência, natureza jurídica do hospital (público ou particular), forma de financiamento da internação (SUS, planos de saúde ou do próprio bolso - out-of-pocket - e a avaliação do atendimento de saúde recebido (muito bom, bom, regular, ruim, muito ruim).

Nas análises multivariadas foram analisadas 17.956 internações referentes às categorias tratamento clínico e cirurgia, correspondendo a $75,1 \%$ das internações. Foram excluídas as internações referentes a parto normal e por cesariana e as internações para tratamento psiquiátrico e realização de exames. Essa exclusão foi feita com o objetivo de tornar o grupo das internações analisadas mais homogêneo.

As variáveis de necessidade empregadas foram: estado de saúde auto-avaliado (muito bom, bom, regular, ruim e muito ruim), restrição de atividades habituais por motivo de saúde (sim ou não), ter estado acamado por motivo de saúde (sim ou não) e presença de doenças crônicas (nenhuma, uma, duas, três e quatro ou mais). O estado funcional dos indivíduos acima de 14 anos foi analisado pela presença ou não de dificuldade para alimentar-se, tomar banho ou ir ao banheiro. As informações sobre restrição de atividade e ter estado acamado referem-se ao período de duas semanas que antecederam a entrevista. As variáveis demográficas analisadas foram idade e sexo; as variáveis sociais utilizadas foram raça auto-referida, anos completos de escolaridade e faixas de renda familiar per capita. A idade foi analisada como variável contínua. Raça foi categorizada em branca e não-branca, esta incluindo preta, amarela, parda e indígena. Os anos de escolaridade foram definidos apenas para indivíduos maiores de 14 anos e agrupados em: sem instrução, de 1 a 4 , de 5 a 8 , e 9 ou mais anos de escolaridade. A renda familiar per capita foi calculada dividindo-se o valor do rendimento mensal familiar pelo número de componentes da família e foi analisada em categorias de salário-mínimo (sem renda, até $0,75 \mathrm{SM}$, de 0,75 a $2 \mathrm{SM}$, mais de 2 SM e sem informação). Quanto aos fatores capacitantes, incluiu-se: ter direito a algum plano de saúde, ter serviço de saúde de procura regular e área de moradia urbana ou rural. Ter um serviço de saúde de uso regular buscou medir 
se a pessoa tinha o hábito de procurar sempre o mesmo profissional ou serviço quando precisava de atendimento de saúde. A eqüidade no uso de serviços hospitalares foi operacionalizada segundo o modelo de Andersen.

O número de não-respostas é bastante baixo na PNAD/1998. Apenas para renda familiar per capita este valor foi maior (3\%), tendo sido criada a categoria "sem informação" nessa variável. Para todas as outras variáveis o número de indivíduos com valores ausentes não ultrapassou $0,5 \%$, excluindo-se estes das análises multivariadas.

As variáveis dependentes dos modelos testados foram: ter estado internado (modelo logístico) e quantas vezes esteve internado nos últimos doze meses, categorizado em nenhuma, uma, duas e três ou mais internações (modelo multinomial). Foram considerados grandes usuários os indivíduos que apresentaram duas ou mais internações no período de um ano.

Todos os modelos testados foram controlados por idade e sexo. Foram ajustados modelos separados para crianças e adultos, devido ao diferente efeito da idade no uso de serviços hospitalares nos dois grupos. O modelo para crianças não incluiu escolaridade e estado funcional. A estratégia de análise envolveu três etapas sucessivas: inicialmente foram incluídas apenas as variáveis de necessidade, depois acrescentaram-se os fatores socioeconômicos e por fim os fatores capacitantes. O processo de escolha do modelo logístico final partiu do modelo completo, do qual foram retiradas sucessivamente variáveis, sendo os modelos reduzidos comparados com o modelo completo através do teste da razão da máxima verossimilhança. Optamos por manter nos modelos multinomiais todas as variáveis presentes nos modelos logísticos, para efeito de comparação.

Pesos foram utilizados para corrigir o fato de que a probabilidade de ter sido selecionado na amostra não era a mesma para todos os indivíduos na população. Foram utilizados pesos normalizados (Lee et al., 1989), de forma a não expandir a amostra. A relação entre o maior e o menor peso é de 6,75. Todas as análises apresentadas são ponderadas. Nas tabelas, os números absolutos em cada categoria de análise são apresentados sem ponderação, com o objetivo de mostrar o real tamanho desses grupos na população estudada. Foram utilizadas técnicas estatísticas apropriadas para a correção do efeito de desenho (Lee et al., 1989). Como os resultados com e sem a correção de efeito de dese- nho são extremamente próximos e levam às mesmas conclusões, são apresentados os resultados sem a correção. Os softwares utilizados foram o SPSS e o SUDAAN.

\section{Resultados}

O coeficiente geral de internação hospitalar no país em 1998 foi de 6,9\%. Os motivos da última internação referida pelo indivíduo foram tratamento clínico (53,8\%); parto $(20,4 \%$, dos quais $41,7 \%$ por cesariana); cirurgia $(21,8 \%)$; tratamento psiquiátrico $(1,7 \%)$ e exames $(2,3 \%)$. O coeficiente de internação clínica foi de 3,8\% e o de cirúrgica foi de 1,5\%. Entre as pessoas que se internaram, 79,7\% o fizeram apenas uma vez; $12,8 \%$, duas vezes; $4,1 \%$, três vezes e $3,4 \%$, quatro ou mais vezes.

Os coeficientes de internação foram maiores para as mulheres $(8,7 \%)$ em comparação aos homens $(5,1 \%)$ e bastante semelhantes para áreas urbanas $(7,0 \%)$ e rurais $(6,7 \%)$. O grupo etário de 80 ou mais anos apresentou o maior coeficiente de internação $(18,7 \%)$ e o grupo etário de 10 a 14 anos o menor (2,4\%). Mulheres de 15 a 39 anos internaram mais, diferença essa que se reduz drasticamente ao se excluir as internações para parto (Figura 1). As pessoas sem renda, bem como as sem escolaridade, apresentaram coeficientes de internação muito maiores do que os dos outros grupos (Tabela 1).

Com relação à última internação no período de referência, $3,7 \%$ das pessoas permaneceram internadas por mais de 30 dias, sendo o tempo médio de permanência (TMP) de 6,3 dias. Os partos tiveram o menor TMP $(3,1$ dias $)$, em relação aos atendimentos clínicos (6,5 dias) e cirúrgicos ( 6,8 dias). A categoria outros, que inclui as internações psiquiátricas, apresentou o maior TMP (16,2 dias) (Tabela 2).

O SUS financiou $63,1 \%$ das internações, e os planos de saúde $24,6 \%$. O atendimento recebido foi considerado muito bom ou bom por $87,3 \%$ dos indivíduos (Tabela 2). Em 13,9\% das internações houve pagamento do próprio bolso de algum valor pela internação. Chamou a atenção o fato de $5,2 \%$ das pessoas que responderam ter se internado pelo SUS informarem ter pago algum valor por essa internação, e que $1,4 \%$ informaram que a última internação foi coberta por algum plano de saúde e também pelo SUS, o que é ilegal. Entre os indivíduos cuja última internação foi coberta por pla- 
Figura 1

Coeficientes de internação hospitalar segundo especialidades clínicas e cirúrgicas, faixa etária e sexo, PNAD/1998.

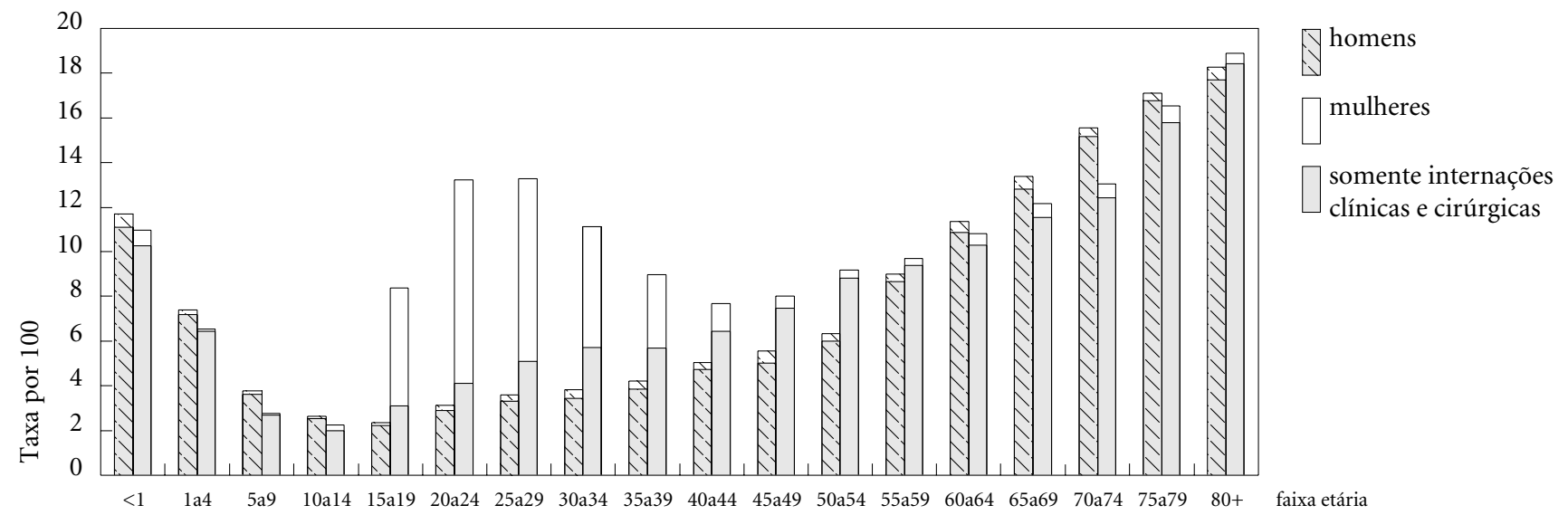

nos pessoas consideraram seu estado de saúde

no de saúde, $15,8 \%$ disseram também ter pago algum valor por essa internação.

Comparando-se os motivos de atendimento, as diferenças mais marcantes foram que $17,0 \%$ de pacientes permaneceram internados por mais de um mês no grupo de outros motivos de internação. Além disso, atendimentos cirúrgicos tiveram maior percentual de internações em estabelecimentos privados, não SUS, cobertos por planos de saúde, nas quais foi pago algum valor pela internação e nas quais o paciente classificou o atendimento recebido como muito bom.

A média de idade foi maior entre os indivíduos que se internaram $(34,6$ anos) em relação aos que não se internaram $(28,0$ anos $)$ e a renda familiar média per capita foi de 246,4 reais e de 254,3 reais, respectivamente. As pessoas que se internaram referiram maior número de doenças crônicas, pior estado de saúde, pior estado funcional, mais restrição das atividades habituais e ter estado acamado por motivo de saúde. Ainda favorável à internação foi dispor de cobertura por plano de saúde e ter um serviço de saúde do qual se faz uso regular. Considerando-se apenas as internações clínicas e cirúrgicas, a média de idade dos que se internaram foi de 37 anos; mais pessoas apresentaram duas ou mais doenças crônicas, tiveram restrição das atividades habituais, estiveram acamadas e apresentavam pior estado funcional e mebom ou muito bom (Tabela 3 ).

Os modelos de uso de serviços de saúde para adultos e crianças são apresentados nas tabelas 4 e 5 respectivamente. Entre os adultos, a cada aumento de um ano na idade observou-se um aumento de $1 \%$ na chance de internação hospitalar, sem diferença quanto ao gênero. A auto-avaliação do estado de saúde foi o fator estudado que mostrou associação mais forte com o uso de serviços hospitalares. Essa variável, a presença de doenças crônicas e o estado funcional apresentaram "efeito dose-resposta" na chance de internação hospitalar. Dentre as variáveis sociais, raça branca esteve associada a uma pequena diferença positiva na chance de internação hospitalar, efeito que se manteve mesmo após a inclusão no modelo dos fatores capacitantes. Ajustada pelas variáveis de necessidades de saúde, a escolaridade se mostrou associada ao uso de serviços hospitalares, apresentando um gradiente desfavorável às pessoas com menos escolaridade. No entanto, a inclusão no modelo dos fatores capacitantes, praticamente anulou o efeito de escolaridade. Com relação à renda, observou-se maior chance de uso para as pessoas mais ricas, no modelo ajustado pelas variáveis de necessidades, mas essa relação se inverteu com a introdução no modelo dos fatores capacitantes. Isto é, as pessoas sem renda e com renda per capita de até 0,75 
Tabela 1

Coeficiente de internação segundo categorias para o período de referência de 12 meses, PNAD 1998.

\begin{tabular}{|c|c|c|c|c|}
\hline \multirow[t]{2}{*}{ Coeficiente de internação } & \multicolumn{2}{|c|}{ Todos os casos } & \multicolumn{2}{|c|}{ Clínicos e cirúrgicos } \\
\hline & $\mathrm{N}$ & Coeficiente & $\mathrm{N}$ & Coeficiente \\
\hline Geral & 23.900 & $6.9 \%$ & 17.956 & $5.3 \%$ \\
\hline \multicolumn{5}{|l|}{ Sexo } \\
\hline Masculino & 8.574 & $5.1 \%$ & 8.080 & $4.9 \%$ \\
\hline Feminino & 15.326 & $8.7 \%$ & 9.876 & $5.8 \%$ \\
\hline Total & 23.900 & & 17.956 & \\
\hline \multicolumn{5}{|l|}{ Faixa etária } \\
\hline Menor de 1 & 724 & $11.1 \%$ & 678 & $10.6 \%$ \\
\hline 1 a 4 & 1.858 & $6.9 \%$ & 1.814 & $6.8 \%$ \\
\hline 5 a 9 & 1.114 & $3.2 \%$ & 1.076 & $3.1 \%$ \\
\hline 10 a 14 & 901 & $2.4 \%$ & 827 & $2.2 \%$ \\
\hline 15 a 19 & 1.997 & $5.4 \%$ & 955 & $2.7 \%$ \\
\hline 20 a 24 & 2.555 & $8.3 \%$ & 1.022 & $3.5 \%$ \\
\hline 25 a 29 & 2.340 & $8.6 \%$ & 1.085 & $4.1 \%$ \\
\hline 30 a 34 & 2.053 & $7.6 \%$ & 1.193 & $4.5 \%$ \\
\hline 35 a 39 & 1.650 & $6.6 \%$ & 1.162 & $4.8 \%$ \\
\hline 40 a 44 & 1.397 & $6.3 \%$ & 1.211 & $5.5 \%$ \\
\hline 45 a 49 & 1.207 & $6.9 \%$ & 1.103 & $6.4 \%$ \\
\hline 50 a 54 & 1.095 & $7.9 \%$ & 1.040 & $7.5 \%$ \\
\hline 55 a 59 & 1.061 & $9.6 \%$ & 1.021 & $9.2 \%$ \\
\hline 60 a 64 & 1.018 & $10.9 \%$ & 967 & $10.5 \%$ \\
\hline 65 a 69 & 942 & $12.6 \%$ & 891 & $12.0 \%$ \\
\hline 70 a 74 & 769 & $14.2 \%$ & 736 & $13.7 \%$ \\
\hline 75 a 79 & 575 & $16.7 \%$ & 552 & $16.0 \%$ \\
\hline 80 ou mais & 642 & $18.7 \%$ & 621 & $18.3 \%$ \\
\hline Total & 23.898 & & 17.954 & \\
\hline \multicolumn{5}{|l|}{ Situação de residência } \\
\hline Urbano & 19.634 & $7.0 \%$ & 14.763 & $5.4 \%$ \\
\hline Rural & 4.266 & $6.7 \%$ & 3.193 & $5.1 \%$ \\
\hline Total & 23.900 & & 17.956 & \\
\hline \multicolumn{5}{|l|}{$\begin{array}{l}\text { Renda familiar per capita } \\
\text { em salários-mínimos }\end{array}$} \\
\hline 0 & 1.151 & $11.5 \%$ & 558 & $6.1 \%$ \\
\hline 0 a $0,75 \mathrm{SM}$ & 9.142 & $7.1 \%$ & 6.534 & $5.2 \%$ \\
\hline 0,75 a 2 SM & 7.690 & $6.7 \%$ & 6.009 & $5.3 \%$ \\
\hline Mais de 2 SM & 5.312 & $6.5 \%$ & 4.366 & $5.4 \%$ \\
\hline Sem informação & 605 & $6.0 \%$ & 489 & $5.0 \%$ \\
\hline Total & 23.900 & & 17.956 & \\
\hline \multicolumn{5}{|l|}{ Escolaridade ( $>14$ anos) } \\
\hline 0 e menos de 1 & 3.617 & $10.4 \%$ & 3.044 & $9.0 \%$ \\
\hline 1 a 4 & 6.173 & $8.7 \%$ & 4.482 & $6.7 \%$ \\
\hline 5 a 8 & 5.114 & $7.0 \%$ & 3.058 & $4.6 \%$ \\
\hline 9 e mais & 4.516 & $6.6 \%$ & 2.926 & $4.4 \%$ \\
\hline Total & 19.420 & & 13.510 & \\
\hline \multicolumn{5}{|l|}{ Raça } \\
\hline Branca & 12.496 & $7.1 \%$ & 9.494 & $5.5 \%$ \\
\hline Outras & 11.404 & $6.7 \%$ & 8.462 & $5.1 \%$ \\
\hline Total & 23.900 & & 17.956 & \\
\hline
\end{tabular}

(Os números absolutos são valores não ponderados e os percentuais são valores ponderados).

${ }^{*}$ Total difere devido a valores ignorados.

Salário-mínimo em 1998: R\$130,00.

Nota: exclusive a população rural de Rondônia, Acre, Amazonas, Roraima, Pará e Amapá. 
Tabela 2

Características da última internação referida pelos indivíduos no período de referência de 12 meses, PNAD/1998.

\begin{tabular}{|c|c|c|c|c|c|c|c|c|c|c|}
\hline \multirow[t]{3}{*}{ Variável } & \multicolumn{10}{|c|}{ Tipo de Tratamento Recebido } \\
\hline & \multicolumn{2}{|c|}{ Clínico } & \multicolumn{2}{|c|}{ Cirurgia } & \multicolumn{2}{|c|}{ Parto $^{\star}$} & \multicolumn{2}{|c|}{ Outros ${ }^{* *}$} & \multicolumn{2}{|c|}{ Total } \\
\hline & $\mathrm{N}$ & $\%$ & $\mathrm{~N}$ & $\%$ & $\mathrm{~N}$ & $\%$ & $\mathrm{~N}$ & $\%$ & $\mathrm{~N}$ & $\%$ \\
\hline $\begin{array}{l}\text { Participação no total } \\
\text { de atendimentos }\end{array}$ & 12.718 & $53.8 \%$ & 5.238 & $21.8 \%$ & 4.964 & $20.4 \%$ & 980 & $4.0 \%$ & 23.900 & $100.0 \%$ \\
\hline \multicolumn{11}{|c|}{$\begin{array}{l}\text { Tempo de permanência (em dias) } \\
\text { (valores ponderados) }\end{array}$} \\
\hline média/desvio-padrão & 6.5 & 12.6 & 6.8 & 13.1 & 3.1 & 4.8 & 16.2 & 33.1 & 6.3 & 13.4 \\
\hline mediana & 4.0 & & 3.0 & & 3.0 & & 4.0 & & 3.0 & \\
\hline \multicolumn{11}{|l|}{ Permanência } \\
\hline$<30$ dias & 12.266 & $96.7 \%$ & 5.000 & $95.6 \%$ & 4.934 & $99.4 \%$ & 816 & $83.0 \%$ & 23.016 & $96.3 \%$ \\
\hline$>=30$ dias & 452 & $3.3 \%$ & 238 & $4.4 \%$ & 30 & $0.6 \%$ & 164 & $17.0 \%$ & 884 & $3.7 \%$ \\
\hline Total & 12.718 & $100.0 \%$ & 5.238 & $100.0 \%$ & 4.964 & $100.0 \%$ & 980 & $100.0 \%$ & 23.900 & $100.0 \%$ \\
\hline \multicolumn{11}{|c|}{ Natureza do estabelecimento } \\
\hline Público & 8.284 & $63.5 \%$ & 2.564 & $47.3 \%$ & 3.418 & $67.8 \%$ & 613 & $60.6 \%$ & 14.879 & $60.8 \%$ \\
\hline Particular & 4.367 & $36.0 \%$ & 2.638 & $52.1 \%$ & 1.524 & $31.9 \%$ & 349 & $37.5 \%$ & 8.878 & $38.7 \%$ \\
\hline Não informado & 67 & $0.5 \%$ & 36 & $0.6 \%$ & 22 & $0.3 \%$ & 18 & $1.9 \%$ & 143 & $0.5 \%$ \\
\hline Total & 12.718 & $100.0 \%$ & 5.238 & $100.0 \%$ & 4.964 & $100.0 \%$ & 980 & $100.0 \%$ & 23.900 & $100.0 \%$ \\
\hline \multicolumn{11}{|l|}{$\begin{array}{l}\text { Pagamento através } \\
\text { de plano de saúde }\end{array}$} \\
\hline Sim & 2.606 & $20.8 \%$ & 2.057 & $40.0 \%$ & 895 & $18.8 \%$ & 215 & $21.8 \%$ & 5.773 & $24.6 \%$ \\
\hline Não & 10.106 & $79.2 \%$ & 3.178 & $59.9 \%$ & 4.068 & $81.2 \%$ & 762 & $77.9 \%$ & 18.114 & $75.4 \%$ \\
\hline Ignorado & 6 & $0.0 \%$ & 3 & $0.1 \%$ & 1 & $0.0 \%$ & 3 & $0.3 \%$ & 13 & $0.0 \%$ \\
\hline Total & 12.718 & $100.0 \%$ & 5.238 & $100.0 \%$ & 4.964 & $100.0 \%$ & 980 & $100.0 \%$ & 23.900 & $100.0 \%$ \\
\hline \multicolumn{11}{|l|}{$\begin{array}{l}\text { Pagamento de algum valor } \\
\text { pela internação }\end{array}$} \\
\hline $\operatorname{Sim}$ & 1.497 & $11.7 \%$ & 1.041 & $20.1 \%$ & 657 & $13.1 \%$ & 139 & $14.5 \%$ & 3.334 & $13.9 \%$ \\
\hline Não & 11.215 & $88.2 \%$ & 4.194 & $79.8 \%$ & 4.306 & $86.9 \%$ & 837 & $85.1 \%$ & 20.552 & $86.0 \%$ \\
\hline Não informado & 6 & $0.1 \%$ & 3 & $0.1 \%$ & 1 & $0.0 \%$ & 4 & $0.4 \%$ & 14 & $0.1 \%$ \\
\hline Total & 12.718 & $100.0 \%$ & 5.238 & $100.0 \%$ & 4.964 & $100.0 \%$ & 980 & $100.0 \%$ & 23.900 & $100.0 \%$ \\
\hline \multicolumn{11}{|l|}{ Internação através do SUS } \\
\hline Sim & 8.642 & $67.8 \%$ & 2.482 & $46.2 \%$ & 3.462 & $69.1 \%$ & 615 & $62.0 \%$ & 15.201 & $63.1 \%$ \\
\hline Não & 3.600 & $28.5 \%$ & 2.603 & $50.9 \%$ & 1.302 & $26.9 \%$ & 314 & $32.6 \%$ & 7.819 & $33.2 \%$ \\
\hline Não Sabe & 476 & $3.7 \%$ & 153 & $2.9 \%$ & 200 & $4.0 \%$ & 51 & $5.4 \%$ & 880 & $3.7 \%$ \\
\hline Total & 12.718 & $100.0 \%$ & 5.238 & $100.0 \%$ & 4.964 & $100.0 \%$ & 980 & $100.0 \%$ & 23.900 & $100.0 \%$ \\
\hline \multicolumn{11}{|l|}{$\begin{array}{l}\text { Avaliação do atendimento } \\
\text { recebido }\end{array}$} \\
\hline Muito bom & 2.924 & $23.0 \%$ & 2.038 & $38.9 \%$ & 1.365 & $27.1 \%$ & 245 & $25.2 \%$ & 6.572 & $27.3 \%$ \\
\hline Bom & 7.876 & $62.3 \%$ & 2.773 & $53.1 \%$ & 2.983 & $61.1 \%$ & 573 & $58.6 \%$ & 14.205 & $60.0 \%$ \\
\hline Regular & 1.479 & $11.4 \%$ & 338 & $6.2 \%$ & 475 & $9.2 \%$ & 118 & $12.2 \%$ & 2.410 & $9.8 \%$ \\
\hline Ruim & 257 & $2.0 \%$ & 46 & $0.9 \%$ & 92 & $1.7 \%$ & 23 & $2.0 \%$ & 418 & $1.7 \%$ \\
\hline Muito ruim & 173 & $1.2 \%$ & 40 & $0.8 \%$ & 47 & $0.8 \%$ & 17 & $1.6 \%$ & 277 & $1.1 \%$ \\
\hline Ignorado & 9 & $0.1 \%$ & 3 & $0.1 \%$ & 2 & $0.1 \%$ & 4 & $0.4 \%$ & 18 & $0.1 \%$ \\
\hline Total & 12.718 & $100.0 \%$ & 5.238 & $100.0 \%$ & 4.964 & $100.0 \%$ & 980 & $100.0 \%$ & 23.900 & $100.0 \%$ \\
\hline
\end{tabular}

(Os números absolutos são valores não ponderados e os percentuais são valores ponderados).

* Inclui parto normal e cesariana.

** Inclui tratamento psiquiátrico, exames e ignorado.

Nota: exclusive a população rural de Rondônia, Acre, Amazonas, Roraima, Pará e Amapá. 
Tabela 3

Características dos indivíduos que foram internados e dos que não foram internados no período de 12 Meses, PNAD/1998.

\begin{tabular}{|c|c|c|c|c|c|c|c|c|}
\hline \multirow[t]{3}{*}{ Variável } & \multicolumn{4}{|c|}{ Todas as Internações } & \multicolumn{4}{|c|}{ Casos Clínicos e Cirúrgicos } \\
\hline & \multicolumn{2}{|c|}{ Internaram } & \multicolumn{2}{|c|}{ Não Internaram } & \multicolumn{2}{|c|}{ Internaram } & \multicolumn{2}{|c|}{ Não Internaram } \\
\hline & $\mathrm{N}$ & $\%$ & $\mathrm{~N}$ & $\%$ & $\mathrm{~N}$ & $\%$ & $\mathrm{~N}$ & $\%$ \\
\hline Idade (em anos) (média e desvio-padrão) & 34.6 & 22.5 & 28.0 & 19.7 & 37.0 & 24.5 & 28.0 & 19.7 \\
\hline $\begin{array}{l}\text { Renda familiar per capita (em reais) } \\
\text { (média e desvio-padrão) }\end{array}$ & 246.4 & 523.7 & 254.3 & 477.0 & 268.3 & 571.5 & 254.3 & 477.0 \\
\hline \multicolumn{9}{|l|}{ Número de doenças crônicas } \\
\hline 0 & 11.116 & $46.5 \%$ & 223.742 & $70.0 \%$ & 7.284 & $40.6 \%$ & 223.742 & $70.0 \%$ \\
\hline 1 & 5.583 & $23.1 \%$ & 56.020 & $17.2 \%$ & 4.310 & $23.7 \%$ & 56.020 & $17.2 \%$ \\
\hline 2 & 2.982 & $12.4 \%$ & 22.829 & $7.1 \%$ & 2.513 & $13.9 \%$ & 22.829 & $7.1 \%$ \\
\hline 3 & 1.960 & $8.2 \%$ & 10.807 & $3.4 \%$ & 1.739 & $9.7 \%$ & 10.807 & $3.4 \%$ \\
\hline 4 ou mais & 2.259 & $9.8 \%$ & 7.565 & $2.3 \%$ & 2.110 & $12.1 \%$ & 7.565 & $2.3 \%$ \\
\hline Total & 23.900 & $100.0 \%$ & 320.963 & & 17.956 & $100.0 \%$ & 320.963 & \\
\hline \multicolumn{9}{|l|}{ Auto-avaliação do estado de saúde } \\
\hline Muito bom & 3.472 & $15.2 \%$ & 88.987 & $29.1 \%$ & 2.057 & $11.8 \%$ & 88.987 & $29.1 \%$ \\
\hline Bom & 9.706 & $40.0 \%$ & 169.285 & $51.7 \%$ & 6.541 & $36.0 \%$ & 169.285 & $51.7 \%$ \\
\hline Regular & 7.539 & $31.5 \%$ & 53.119 & $16.2 \%$ & 6.496 & $36.3 \%$ & 53.119 & $16.2 \%$ \\
\hline Ruim & 2.505 & $10.6 \%$ & 7.920 & $2.5 \%$ & 2.254 & $12.7 \%$ & 7.920 & $2.5 \%$ \\
\hline Muito ruim & 674 & $2.7 \%$ & 1.575 & $0.5 \%$ & 604 & $3.2 \%$ & 1.575 & $0.5 \%$ \\
\hline Ignorado & 4 & $0.0 \%$ & 77 & $0.0 \%$ & 4 & $0.0 \%$ & 77 & $0.0 \%$ \\
\hline Total & 23.900 & $100.0 \%$ & 320.963 & $100.0 \%$ & 17.956 & $100.0 \%$ & 320.963 & $100.0 \%$ \\
\hline \multicolumn{9}{|l|}{ Restrição de atividades habituais ${ }^{*}$} \\
\hline Sim & 5.099 & $21.3 \%$ & 17.295 & $5.2 \%$ & $4 . .462$ & $24.7 \%$ & 17.295 & $5.2 \%$ \\
\hline Não & 18.801 & $78.7 \%$ & 303.668 & $94.8 \%$ & 13.494 & $75.3 \%$ & 303.668 & $94.8 \%$ \\
\hline Total & 23.900 & $100.0 \%$ & 320.963 & $100.0 \%$ & 17.956 & $100.0 \%$ & 320.963 & $100.0 \%$ \\
\hline \multicolumn{9}{|l|}{ Ter estado acamado* } \\
\hline Sim & 3.832 & $16.2 \%$ & 9.976 & $3.0 \%$ & 4.462 & $19.0 \%$ & 9.976 & $3.0 \%$ \\
\hline Não & 20.067 & $83.8 \%$ & 310.979 & $97.0 \%$ & 13.494 & $81.0 \%$ & 310.979 & $97.0 \%$ \\
\hline Ignorado & 1 & $0.0 \%$ & 8 & $0.0 \%$ & 0 & $0.0 \%$ & 8 & $0.0 \%$ \\
\hline Total & 23.900 & $100.0 \%$ & 320.963 & $100.0 \%$ & 17.956 & $100.0 \%$ & 320.963 & $100.0 \%$ \\
\hline \multicolumn{9}{|l|}{ Cobertura por plano de saúde } \\
\hline Um plano & 6.155 & $26.0 \%$ & 71.369 & $22.5 \%$ & 4.911 & $27.5 \%$ & 71.369 & $22.5 \%$ \\
\hline Mais de 1 plano & 612 & $2.3 \%$ & 6.275 & $1.7 \%$ & 490 & $2.5 \%$ & 6.275 & $1.7 \%$ \\
\hline Não tem direito & 17.133 & $71.7 \%$ & 243.308 & $75.8 \%$ & 12.555 & $70.0 \%$ & 243.308 & $75.8 \%$ \\
\hline Ignorado & 0 & $0.0 \%$ & 11 & $0.0 \%$ & 0 & $0.0 \%$ & 11 & $0.0 \%$ \\
\hline Total & 23.900 & $100.0 \%$ & 320.963 & $100.0 \%$ & 17.956 & $100.0 \%$ & 320.963 & $100.0 \%$ \\
\hline \multicolumn{9}{|l|}{ Serviço de saúde de procura regular } \\
\hline Sim & 18.567 & $78.6 \%$ & 244.661 & $70.7 \%$ & 13.951 & $78.6 \%$ & 244.661 & $70.7 \%$ \\
\hline Não & 5.333 & $21.4 \%$ & 96.295 & $29.3 \%$ & 4.005 & $21.4 \%$ & 96.295 & $29.3 \%$ \\
\hline Ignorado & 0 & $0.0 \%$ & 7 & $0.0 \%$ & 0 & $0.0 \%$ & 7 & $0.0 \%$ \\
\hline Total & 23.900 & $100.0 \%$ & 340.963 & $100.0 \%$ & 17.956 & $100.0 \%$ & 340.963 & $100.0 \%$ \\
\hline \multicolumn{9}{|l|}{$\begin{array}{l}\text { Dificuldade para alimentar-se, } \\
\text { tomar banho e ir ao banheiro }\end{array}$} \\
\hline Não tem dificuldade & 17.177 & $72.0 \%$ & 221.349 & $69.1 \%$ & 11.615 & $65.1 \%$ & 221.349 & $69.1 \%$ \\
\hline Pequena dificuldade & 1.216 & $5.3 \%$ & 5.042 & $1.6 \%$ & 1.097 & $6.3 \%$ & 5.042 & $1.6 \%$ \\
\hline Grande dificuldade & 760 & $3.2 \%$ & 1.673 & $0.5 \%$ & 686 & $3.8 \%$ & 1.673 & $0.5 \%$ \\
\hline Não consegue & 329 & $1.4 \%$ & 654 & $0.2 \%$ & 306 & $1.7 \%$ & 654 & $0.2 \%$ \\
\hline Não se aplica ${ }^{\star \star}$ & 4.418 & $18.1 \%$ & 92.207 & $28.6 \%$ & 4.252 & $23.1 \%$ & 92.207 & $28.6 \%$ \\
\hline Ignorado & 0 & $0.0 \%$ & 38 & $0.0 \%$ & 0 & $0.0 \%$ & 38 & $0.0 \%$ \\
\hline Total & 23.900 & $100.0 \%$ & 320.963 & $100.0 \%$ & 17.956 & $100.0 \%$ & 320.963 & $100.0 \%$ \\
\hline
\end{tabular}

(Os números absolutos são valores não ponderados e os percentuais são valores ponderados).

${ }^{*}$ No período de referência de quinze dias anteriores à pesquisa.

** Pessoas menores de 14 anos para as quais essa informação não foi coletada.

Nota: exclusive a população rural de Rondônia, Acre, Amazonas, Roraima, Pará e Amapá. 
salários-mínimos passaram a apresentar maior chance de internação hospitalar. Ajustado pelas demais variáveis, os adultos que tinham plano de saúde e serviço de saúde de uso regular apresentaram maior chance de internações hospitalares, sendo o efeito de ter mais de um plano ainda maior (Tabela 4).

Nas crianças, a cada aumento de um ano de idade observou-se uma diminuição de 13\% na chance de internação hospitalar, com maior chance de internação para o sexo masculino. Como observado nos adultos, a auto-avaliação do estado de saúde foi o fator com o maior associação com o uso de serviços hospitalares. Essa variável, bem como a presença de doenças crônicas, também apresentou "efeito dose-resposta”. Como nos adultos, as crianças sem renda e com renda familiar per capita de até 0,75 salários-mínimos apresentaram maior chance de internação hospitalar, somente depois da inclusão no modelo dos fatores capacitantes. Ajustado pelas demais variáveis, as crianças que tinham plano de saúde e serviço de saúde de uso regular apresentaram maior chance de se internar, porém ter mais de um plano de saúde não afetou as chances de internação hospitalar nesse grupo (Tabela 5).

No caso de internações múltiplas nos adultos, foram observadas variações entre o grupo que referiu duas internações e aquele com três ou mais internações, em relação ao grupo com uma única internação. As pessoas que referiram duas internações no período diferiram pouco daquelas com apenas uma internação. Não houve variação em relação ao sexo e a idade. No geral, as condições de saúde foram um pouco piores, mas não se observaram diferenças em relação às condições sociais e aos fatores capacitantes. A única exceção foi em relação às pessoas que referiram ter serviço de uso regular, que se mostraram mais propensas a uma segunda internação do que as pessoas que referiram não ter serviço de uso regular. Por outro lado, as pessoas com três ou mais internações, diferiram de forma mais expressiva do grupo com uma internação nas variáveis de necessidades, isto é, apresentaram condições de saúde bem piores. Diferiram também nos fatores sociais, pois se concentraram nos grupos de menor renda e menor escolaridade. Mas não se observou variação nas chances de mais de uma internação hospitalar em relação à raça (Tabela 6).

No caso de internações múltiplas entre as crianças, os resultados foram semelhantes aos observados nos adultos. A magnitude do efeito das variáveis de necessidade também foi maior no grupo com três ou mais internações. Não se observou variação em relação à renda no grupo com duas internações, mas no grupo que apresentou três ou mais internações, as pessoas de menor renda apresentaram maior chance de se internar (Tabela 7). O risco de uma segunda internação variou inversamente com a idade, mas não variou no caso de três ou mais internações. $\mathrm{O}$ sexo não se mostrou associado ao risco de mais de uma internação hospitalar nas crianças.

\section{Discussão}

Como se esperava, a necessidade de saúde foi o fator explicativo mais importante na utilização de serviços hospitalares no Brasil. Como necessidade de saúde é um fenômeno incomensurável, foram utilizadas mais do que uma variável de estado de saúde como proxies de necessidade. Mesmo assim, considera-se que parte da necessidade de saúde permaneceu sem ser medida. Os indicadores de necessidade de saúde utilizados foram baseados em morbidade referida e, dessa forma, diferem do estado de saúde obtido a partir de avaliação clínica.

Foram observadas desigualdades sociais no consumo de serviços hospitalares no Brasil, em 1998. Ao ajustarmos por necessidade de saúde, observamos uma desigualdade pequena, porém desfavorável às pessoas em piores condições sociais. A direção dessa desigualdade se inverte quando ajustamos também pelos fatores capacitantes (plano de saúde e serviço de uso regular). Isto é, no modelo ajustado por necessidade de saúde e fatores capacitantes, as pessoas com menor renda apresentaram maior chance de se internar; e no modelo ajustado somente por necessidade de saúde as pessoas com maior renda apresentaram maior chance de se internar. Efeito semelhante ao observado para renda ocorreu no caso da educação entre adultos. Pelo menos uma hipótese pode ser considerada na interpretação desses resultados: ter plano de saúde ou ter um serviço de uso regular aumentaram as chances de uso das pessoas de pior condição social. Isto é, em condições de igualdade desses fatores, as pessoas mais pobres conseguiram superar restrições para internação hospitalar impostas pela sua condição social.

Manteve-se uma pequena desigualdade no uso de serviços hospitalares em favor da raça branca nos adultos, mesmo após o ajuste por 
Tabela 4

Modelos explicativos da utilização de internações hospitalares no Brasil para adultos (mais de 14 anos), PNAD/1998.

\begin{tabular}{|c|c|c|c|c|c|c|}
\hline \multirow{3}{*}{$\begin{array}{l}\text { N (não ponderado) } \\
\text { Variável }\end{array}$} & \multicolumn{2}{|c|}{ Modelo Necessidade } & \multicolumn{2}{|c|}{ Necessidade e Sociais } & \multicolumn{2}{|c|}{ Modelo Completo } \\
\hline & \multicolumn{2}{|c|}{$234.914 \begin{array}{c}125 \\
\text { ignorados }\end{array}$} & \multicolumn{2}{|c|}{$\begin{array}{rc}233.709 & 1.330 \\
& \text { ignorados }\end{array}$} & 233.699 & $\begin{array}{c}1.340 \\
\text { ignorados }\end{array}$ \\
\hline & OR & IC $95 \%$ & OR & IC $95 \%$ & OR & IC $95 \%$ \\
\hline \multicolumn{7}{|l|}{ Idade } \\
\hline a cada ano & 1.01 & $1,01-1,01$ & 1.01 & $1,01-1,01$ & 1.01 & $1,01-1,01$ \\
\hline \multicolumn{7}{|l|}{ Sexo } \\
\hline Masculino & 1.00 & & 1.00 & & 1.00 & \\
\hline Feminino & 1.07 & $1,04-1,11$ & 1.07 & $1,03-1,11$ & 1.04 & $1,00-1,08$ \\
\hline \multicolumn{7}{|c|}{ Auto-avaliação estado de saúde } \\
\hline Muito bom & 1.00 & & 1.00 & & 1.00 & \\
\hline Bom & 1.50 & $1,40-1,60$ & 1.54 & $1,44-1,65$ & 1.54 & $1,44-1,64$ \\
\hline Regular & 2.82 & $2,62-3,03$ & 3.00 & $2,79-3,23$ & 3.04 & $2,82-3,27$ \\
\hline Ruim & 3.81 & $3,48-4,16$ & 4.17 & $3,81-4,58$ & 4.28 & $3,90-4,70$ \\
\hline Muito ruim & 3.86 & $3,38-4,41$ & 4.20 & $3,68-4,81$ & 4.35 & $3,80-4,98$ \\
\hline \multicolumn{7}{|c|}{ Restrição das atividades habituais } \\
\hline Não & 1.00 & & 1.00 & & 1.00 & \\
\hline Sim & 1.79 & $1,68-1,92$ & 1.78 & $1,67-1,90$ & 1.75 & $1,64-1,87$ \\
\hline \multicolumn{7}{|l|}{ Estiveram acamados } \\
\hline Não & 1.00 & & 1.00 & & 1.00 & \\
\hline Sim & 2.11 & $1,96-2,27$ & 2.12 & $1,98-2,28$ & 2.13 & $1,98-2,29$ \\
\hline \multicolumn{7}{|c|}{ Número de doenças crônicas } \\
\hline Nenhuma & 1.00 & & 1.00 & & 1.00 & \\
\hline Uma & 1.57 & $1,48-1,67$ & 1.57 & $1,48-1,67$ & 1.55 & $1,45-1,65$ \\
\hline Duas & 1.44 & $1,37-1,51$ & 1.43 & $1,36-1,51$ & 1.41 & $1,34-1,48$ \\
\hline Três & 1.80 & $1,68-1,93$ & 1.81 & $1,68-1,94$ & 1.77 & $1,65-1,90$ \\
\hline Quatro ou mais & 2.30 & $2,14-2,48$ & 2.31 & $2,14-2,49$ & 2.27 & $2,10-2,44$ \\
\hline \multicolumn{7}{|c|}{$\begin{array}{l}\text { Dificuldade para alimentar-se, } \\
\text { tomar banho e ir ao banheiro }\end{array}$} \\
\hline Não tem dificuldade & 1.00 & & 1.00 & & 1.00 & \\
\hline Pequena dificuldade & 1.41 & $1,31-1,52$ & 1.44 & $1,34-1,55$ & 1.46 & $1,35-1,57$ \\
\hline Grande dificuldade & 1.68 & $1,52-1,86$ & 1.72 & $1,55-1,91$ & 1.74 & $1,57-1,93$ \\
\hline Não consegue & 1.75 & $1,51-2,05$ & 1.77 & $1,51-2,07$ & 1.80 & $1,54-2,10$ \\
\hline \multicolumn{7}{|c|}{$\begin{array}{l}\text { Renda familiar per capita } \\
\text { em faixas de salário-mínimo }\end{array}$} \\
\hline Mais de 2 SM & & & 1.00 & & 1.00 & \\
\hline 0,75 a $2 \mathrm{SM}$ & & & 0.87 & $0,83-0,91$ & 1.01 & $0,96-1,06^{*}$ \\
\hline Até $0,75 \mathrm{SM}$ & & & 0.91 & $0,86-0,96$ & 1.12 & $1,06-1,19$ \\
\hline Sem renda & & & 1.02 & $0,89-1,17^{\star}$ & 1.27 & $1,11-1,46$ \\
\hline Sem informação & & & 0.95 & $0,85-1,05^{\star}$ & 1.04 & $0,93-1,16^{*}$ \\
\hline \multicolumn{7}{|l|}{ Escolaridade } \\
\hline 9 ou mais anos & & & 1.00 & & 1.00 & \\
\hline 5 a 8 anos & & & 0.95 & $0,87-0,98$ & 1.05 & $1,00-1,13$ \\
\hline 1 a 4 anos & & & 0.92 & $0,89-1,00$ & 1.06 & $0,99-1,11^{*}$ \\
\hline Sem instrução & & & 0.83 & $0,77-0,89$ & 0.99 & $0,93-1,07^{\star}$ \\
\hline
\end{tabular}

(continua) 
Tabela 4

(continuação)

\begin{tabular}{|c|c|c|c|c|c|c|}
\hline \multirow{3}{*}{$\begin{array}{l}\text { N (não ponderado) } \\
\text { Variável }\end{array}$} & \multicolumn{2}{|c|}{ Modelo Necessidade } & \multicolumn{2}{|c|}{ Necessidade e Sociais } & \multicolumn{2}{|c|}{ Modelo Completo } \\
\hline & \multicolumn{2}{|c|}{ ignorados } & \multicolumn{2}{|c|}{ ignorados } & \multicolumn{2}{|c|}{ ignorados } \\
\hline & OR & IC 95\% & OR & IC 95\% & OR & IC 95\% \\
\hline \multicolumn{7}{|l|}{ Raça } \\
\hline Outros & & & 1.00 & & 1.00 & \\
\hline Brancos & & & 1.10 & $1,06-1,14$ & 1.06 & $1,02-1,11$ \\
\hline \multicolumn{7}{|l|}{ Plano de saúde } \\
\hline Não tem & & & & & 1.00 & \\
\hline Um & & & & & 1.61 & $1,53-1,69$ \\
\hline Mais de um & & & & & 2.37 & $2,11-2,67$ \\
\hline \multicolumn{7}{|c|}{ Serviço de saúde de uso regular } \\
\hline Não & & & & & 1.00 & \\
\hline Sim & & & & & 1.27 & $1,22-1,33$ \\
\hline
\end{tabular}

* Não significativo

Salário-mínimo em 1998: R\$130,00.

Nota 1: exclusive a população rural de Rondônia, Acre, Amazonas, Roraima, Pará e Amapá.

Nota 2: exclusive parto normal, cesariana, tratamento psiquiátrico e realização de exames.

necessidade de saúde e fatores capacitantes. Como raça é uma classificação baseada na cor da pele, mas que mede aspectos das relações sociais (Jones, 2001), esse resultado sugere que pode ter ocorrido, mesmo que em reduzida freqüência, seleção desfavorável aos não brancos na decisão de internar. A discriminação atuaria diminuindo, para os brasileiros não brancos e pobres, o efeito compensatório nas desigualdades no uso de serviços de saúde que o hospital aparentemente tem entre as pessoas de menor renda no Brasil.

Pesquisa feita comparando-se o uso de serviços de saúde entre diferentes áreas geográficas e diferentes grupos sociais no Brasil mostrou que os grupos de mais alta renda consomem mais serviços ambulatoriais, que o consumo de serviços hospitalares tende a aumentar à medida que a renda decresce e que houve um aumento importante na participação do hospital (incluindo internação, pronto-socorro e ambulatório hospitalar) na cesta de consumo de serviços de saúde no Brasil entre 1989 e 1996 (Travassos et al., 2000). Almeida et al. (2000) mostraram que as desigualdades observadas no consumo de serviços hospitalares eram bem menos acentuadas do que as desigualdades observadas no consumo de serviços de saúde em geral no Brasil. Nunes et al. (2001) também observaram que as taxas brutas de internações no Brasil eram praticamente invariá- veis entre diferentes grupos de renda; que as desigualdades regionais no consumo de internações hospitalares no Brasil diminuíram com a implantação do SUS e que essas desigualdades eram menores do que as desigualdades no consumo de outros serviços de saúde.

É possível que as respostas às perguntas sobre morbidade auto-referida tenham variado entre os grupos sociais. No caso de pessoas de pior condição social terem menor tendência a declarar morbidade do que as pessoas de melhor condição social seria introduzido um erro que iria resultar em pior ajuste por necessidade entre as pessoas com pior condição social. Nesse caso, a diferença em favor dos ricos seria um artefato dos dados e a diferença no modelo final em favor das pessoas com pior condição social seria ainda maior. No entanto, recente estudo (Eriksson et al., 2001) realizado na Suécia não identificou variação social importante nas respostas a diferentes perguntas de auto-avaliação do estado de saúde.

Algumas informações da PNAD/1998, como o número de internações, são referidas a um período maior, de doze meses, enquanto outras, como a restrição de atividades habituais e ter estado acamado, dizem respeito a duas semanas. Essas diferentes referências temporais podem ter tido influência sobre os resultados encontrados. A PNAD/1998 não permite analisar as pessoas que precisaram mas não usaram 
Tabela 5

Modelos explicativos da utilização de internações hospitalares no Brasil para crianças (até 14 anos), PNAD/1998.

\begin{tabular}{|c|c|c|c|c|c|c|}
\hline \multirow{4}{*}{$\begin{array}{l}\text { N (não ponderado) } \\
\text { Variável }\end{array}$} & \multicolumn{2}{|c|}{ Modelo Necessidade } & \multicolumn{2}{|c|}{ Necessidade e Sociais } & \multicolumn{2}{|c|}{ Modelo Completo } \\
\hline & \multirow{2}{*}{\multicolumn{2}{|c|}{$\begin{array}{rc}103.811 & 36 \\
\text { ignorados }\end{array}$}} & \multirow{2}{*}{\multicolumn{2}{|c|}{$103.811 \underset{ }{36} \begin{array}{c}36 \\
\text { ignorados }\end{array}$}} & \multirow{2}{*}{\multicolumn{2}{|c|}{$\begin{array}{lc}103.803 & 44 \\
\text { ignorados }\end{array}$}} \\
\hline & & & & & & \\
\hline & OR & IC 95\% & OR & IC 95\% & OR & IC 95\% \\
\hline \multicolumn{7}{|l|}{ Idade } \\
\hline a cada ano & 0.87 & $0,86-0,88$ & 0.87 & $0,86-0,88$ & 0.87 & $0,86-0,88$ \\
\hline \multicolumn{7}{|l|}{ Sexo } \\
\hline Masculino & 1.00 & & 1.00 & & 0.86 & $0,81-0,92$ \\
\hline Feminino & 0.86 & $0,81-0,92$ & 0.86 & $0,81-0,92$ & & \\
\hline \multicolumn{7}{|c|}{ Auto-avaliação estado de saúde } \\
\hline Muito bom & 1.00 & & 1.00 & & 1.00 & \\
\hline Bom & 1.56 & $1,45-1,69$ & 1.58 & $1,46-1,70$ & 1.56 & $1,45-1,69$ \\
\hline Regular & 3.46 & $3,12-3,84$ & 3.52 & $3,16-3,91$ & 3.54 & $3,18-3,93$ \\
\hline Ruim e muito ruim & 5.62 & $4,59-6,88$ & 5.72 & $4,67-7,02$ & 5.81 & $4,73-7,12$ \\
\hline \multicolumn{7}{|c|}{ Restrição de atividades habituais } \\
\hline Não & 1.00 & & 1.00 & & 1.00 & \\
\hline Sim & 1.58 & $1,39-1,81$ & 1.58 & $1,38-1,80$ & 1.55 & $1,35-1,77$ \\
\hline \multicolumn{7}{|l|}{ Estiveram acamados } \\
\hline Não & 1.00 & & 1.00 & & 1.00 & \\
\hline Sim & 1.94 & $1,67-2,26$ & 1.94 & $1,67-2,26$ & 1.95 & $1,68-2,27$ \\
\hline \multicolumn{7}{|c|}{ Número de doenças crônicas } \\
\hline Nenhuma & 1.00 & & 1.00 & & 1.00 & \\
\hline Uma & 2.35 & $2,16-2,55$ & 2.34 & $2,16-2,55$ & 2.29 & $2,11-2,49$ \\
\hline Duas ou mais & 3.18 & $2,50-4,04$ & 3.16 & $2,49-4,02$ & 3.07 & $2,41-3,90$ \\
\hline \multicolumn{7}{|c|}{$\begin{array}{l}\text { Renda familiar per capita } \\
\text { em faixas de salário-mínimo }\end{array}$} \\
\hline Mais de 2 SM & & & 1.00 & & 1.00 & \\
\hline 0,75 a 2 SM & & & 0.84 & $0,76-0,94$ & 0.96 & $0,86-1,08^{\star}$ \\
\hline Até 0,75 SM & & & 0.87 & $0,80-0,97$ & 1.13 & $1,01-1,26$ \\
\hline Sem renda & & & 0.95 & $0,81-1,11^{\star}$ & 1.20 & $1,02-1,42$ \\
\hline Sem informação & & & 0.81 & $0,62-1,04^{*}$ & 0.95 & $0,73-1,24^{*}$ \\
\hline \multicolumn{7}{|l|}{ Plano de saúde } \\
\hline Não tem & & & & & 1.00 & \\
\hline Um & & & & & 1.46 & $1,34-1,59$ \\
\hline Mais de um & & & & & 1.25 & $0,94-1,65^{*}$ \\
\hline \multicolumn{7}{|c|}{ Serviço de saúde de uso regular } \\
\hline Não & & & & & 1.00 & \\
\hline Sim & & & & & 1.28 & $1,18-1,38$ \\
\hline
\end{tabular}

* Não significativo

Salário-mínimo em 1998: R \$130,00.

Nota 1: exclusive a população rural de Rondônia, Acre, Amazonas, Roraima, Pará e Amapá.

Nota 2: exclusive parto normal, cesariana, tratamento psiquiátrico e realização de exames. 
Tabela 6

Modelos explicativos da utilização de internações hospitalares no Brasil para adultos (mais de 14 anos), PNAD/1998. (Categoria de referência: indivíduos com uma internação)

\begin{tabular}{|c|c|c|c|c|}
\hline \multirow[t]{2}{*}{ Variável } & \multicolumn{2}{|c|}{ Três ou mais } & \multicolumn{2}{|c|}{ Duas internações } \\
\hline & OR & IC 95\% & OR & IC $95 \%$ \\
\hline \multicolumn{5}{|l|}{ Idade } \\
\hline a cada ano & 1.00 & $0,99-1,00$ & 1.00 & $1,00-1,00^{\star}$ \\
\hline \multicolumn{5}{|l|}{ Sexo } \\
\hline Masculino & 1.00 & & 1.00 & \\
\hline Feminino & 0.92 & $0,82-1,05^{\star}$ & 0.98 & $0,89-1,08^{\star}$ \\
\hline \multicolumn{5}{|c|}{ Auto-avaliação estado de saúde } \\
\hline Muito bom & 1.00 & & 1.00 & \\
\hline Bom & 1.03 & $0,70-1,52^{\star}$ & 0.97 & $0,78-1,20^{*}$ \\
\hline Regular & 2.38 & $1,63-3,47$ & 1.40 & $1,13-1,75$ \\
\hline Ruim & 3.88 & $2,60-5,79$ & 1.81 & $1,41-2,32$ \\
\hline Muito ruim & 5.40 & $3,47-8,41$ & 2.18 & $1,59-2,98$ \\
\hline \multicolumn{5}{|c|}{ Número de doenças crônicas } \\
\hline Nenhuma & 1.00 & & 1.00 & \\
\hline Uma & 1.27 & $1,02-1,58$ & 1.19 & $1,03-1,38$ \\
\hline Duas & 1.67 & $1,33-2,10$ & 1.51 & $1,29-1,78$ \\
\hline Três & 1.66 & $1,30-2,11$ & 1.45 & $1,21-1,74$ \\
\hline Quatro ou mais & 2.59 & $2,06-3,27$ & 1.72 & $1,43-2,04$ \\
\hline \multicolumn{5}{|c|}{$\begin{array}{l}\text { Dificuldade para alimentar-se, } \\
\text { tomar banho e ir ao banheiro }\end{array}$} \\
\hline Não tem & 1.00 & & 1.00 & \\
\hline Pequena dificuldade & 1.51 & $1,25-1,82$ & 1.45 & $1,23-1,70$ \\
\hline Grande dificuldade & 1.45 & $1,15-1,82$ & 1.47 & $1,20-1,81$ \\
\hline Não consegue & 2.01 & $1,48-2,73$ & 1.60 & $1,19-2,15$ \\
\hline \multicolumn{5}{|l|}{ Estiveram acamados } \\
\hline Não & 1.00 & & 1.00 & \\
\hline Sim & 1.52 & $1,27-1,85$ & 1.12 & $0,96-1,33^{\star}$ \\
\hline \multicolumn{5}{|c|}{ Restrição de atividades habituais } \\
\hline Não & 1.00 & & 1.00 & \\
\hline Sim & 1.08 & $0,90-1,30^{*}$ & 1.04 & $0,89-1,21^{\star}$ \\
\hline \multicolumn{5}{|c|}{$\begin{array}{l}\text { Renda familiar per capita } \\
\text { em faixas de salário-mínimo }\end{array}$} \\
\hline Mais de 2 SM & 1.00 & & 1.00 & \\
\hline 0,75 a 2 SM & 1.27 & $1,06-1,52$ & 1.07 & $0,94-1,23^{*}$ \\
\hline Até $0,75 \mathrm{SM}$ & 1.27 & $1,03-1,56$ & 1.16 & $0,99-1,35^{\star}$ \\
\hline Sem renda & 2.11 & $1,42-3,14$ & 1.21 & $0,84-1,75^{\star}$ \\
\hline Sem informação & 1.15 & $0,77-1,71^{\star}$ & 0.80 & $0,58-1,11^{\star}$ \\
\hline \multicolumn{5}{|l|}{ Escolaridade } \\
\hline 9 ou mais anos & 1.00 & & 1.00 & \\
\hline 5 a 8 anos & 1.16 & $0,92-1,48^{*}$ & 1.06 & $0,90-1,25^{\star}$ \\
\hline 1 a 4 anos & 1.32 & $1,06-1,66$ & 1.09 & $0,93-1,28^{\star}$ \\
\hline Sem instrução & 1.60 & $1,24-2,06$ & 1.09 & $0,90-1,31^{\star}$ \\
\hline
\end{tabular}

(continua) 
Tabela 6

(continuação)

\begin{tabular}{|c|c|c|c|c|}
\hline \multirow[t]{2}{*}{ Variável } & \multicolumn{2}{|c|}{ Três ou mais } & \multicolumn{2}{|c|}{ Duas internações } \\
\hline & OR & IC 95\% & OR & IC 95\% \\
\hline \multicolumn{5}{|l|}{ Raça } \\
\hline Brancos & 1.00 & & 1.00 & \\
\hline Outros & 0.95 & $0,84-1,08^{\star}$ & 0.97 & $0,87-1,07^{\star}$ \\
\hline \multicolumn{5}{|l|}{ Plano de saúde } \\
\hline Não tem & 1.00 & & 1.00 & \\
\hline Um & 1.18 & $1,01-1,39$ & 1.06 & $0,94-1,21^{\star}$ \\
\hline Mais de um & 0.51 & $0,27-0,98$ & 0.96 & $0,70-1,33^{*}$ \\
\hline \multicolumn{5}{|c|}{ Serviço de saúde de uso regular } \\
\hline Não & 1.00 & & 1.00 & \\
\hline Sim & 1.12 & $0,97-1,31^{\star}$ & 1.13 & $1,00-1,27$ \\
\hline
\end{tabular}

* Não significativo.

Salário-mínimo em 1998: R\$130,00.

Nota 1: exclusive a população rural de Rondônia, Acre, Amazonas, Roraima, Pará e Amapá.

Nota 2: exclusive parto normal, cesariana, tratamento psiquiátrico e realização de exames.

serviços de hospital, e os motivos desse não uso. Apesar de a PNAD ter incluído questões sobre parte da procura de serviços de saúde que não resultou em uso efetivo, não é possível separar qual parte dessa demanda não atendida teria resultado em admissões hospitalares.

A pouca importância relativa das medidas de renda e educação na explicação da ocorrência de internações hospitalares, após ajuste por necessidade de saúde, foi inesperado, considerando um país tão marcado por desigualdades, como é o Brasil, mesmo reconhecendo que parte do efeito da situação social se explicita pela necessidade de saúde. Além disso, os resultados encontrados para grupos de renda parecem estar em desacordo com a "Inverse Care Law", proposta por Hart em 1971.

A "Inverse Care Law" pode ser resumida da seguinte forma: a disponibilidade de cuidado médico de boa qualidade tende a variar inversamente com a necessidade desse cuidado na população atendida; essa lei opera de forma mais completa quando o cuidado médico é exposto às forças de mercado, e menos completa quando essa exposição é reduzida (Hart, 2000). Uma extensão dessa pesquisa será incorporar a dimensão da oferta de serviços de saúde às análises, de forma a verificar como a oferta de serviços afeta os resultados encontrados neste trabalho.

Escolher categorias de renda num país de marcadas desigualdades inter-regionais, como o Brasil, é bastante difícil. Uma categorização por quintis ou decis de renda, por exemplo, teria a desvantagem de agrupar os indivíduos das regiões mais pobres nas faixas inferiores e os indivíduos das regiões mais ricas nas superiores. Após análises exploratórias incluindo o uso de quintis e decis, optou-se por trabalhar com quatro categorias de renda ressaltando as diferenças de renda existentes no extremo inferior da distribuição de renda no país, conforme sugestão baseada em outras análises dos dados da própria PNAD (Viacava et al., 2001).

A chance de internações múltiplas no período estudado também foi fortemente associada à necessidade de saúde. No entanto, todas as variáveis de necessidade mostraram-se menos relacionadas ao uso nas pessoas com duas internações, em comparação com aquelas com mais do que duas internações. Além disso, não houve associação entre variáveis sociais e ocorrência de duas internações, porém houve associação entre essas variáveis e a ocorrência de mais do que duas internações. Esses resultados parecem indicar que existe uma associação entre a primeira e a segunda internação, isto é, que a segunda internação seria predominantemente decorrência da primeira, ou seja, uma reinternação, no sentido mais estrito do termo. O principal determinante de uma segunda internação, indicado por esta análise, seria o estado de saúde do paciente. Os fatores sociais seriam irrelevantes. Entretanto, uma terceira 
Tabela 7

Modelos explicativos da utilização de internações hospitalares no Brasil para crianças (menos de 14 anos), PNAD/1998. (Categoria de referência: indivíduos com uma internação).

\begin{tabular}{|c|c|c|c|c|}
\hline \multirow[t]{2}{*}{ Variável } & \multicolumn{2}{|c|}{ Três ou mais } & \multicolumn{2}{|c|}{ Duas internações } \\
\hline & OR & IC 95\% & OR & IC 95\% \\
\hline \multicolumn{5}{|l|}{ Idade } \\
\hline a cada ano & 0.98 & $0,95-1,01^{*}$ & 0.97 & 0,94-0,99 \\
\hline \multicolumn{5}{|l|}{ Sexo } \\
\hline Masculino & 1.00 & & 1.00 & \\
\hline Feminino & 0.96 & $0,74-1,23^{*}$ & 0.97 & $0,81-1,17^{\star}$ \\
\hline \multicolumn{5}{|c|}{ Auto-avaliação estado de saúde } \\
\hline Muito bom & 1.00 & & 1.00 & \\
\hline Bom & 1.41 & $0,89-2,26^{*}$ & 1.37 & $1,05-1,79$ \\
\hline Regular & 4.43 & $2,75-7,14$ & 2.28 & $1,68-3,10$ \\
\hline Ruim ou muito ruim & 10.62 & $5,91-19,10$ & 3.33 & $2,02-5,47$ \\
\hline \multicolumn{5}{|c|}{ Número de doenças crônicas } \\
\hline Nenhuma & 1.00 & & 1.00 & \\
\hline Uma & 2.13 & $1,61-2,81$ & 1.65 & $1,34-2,05$ \\
\hline Duas ou mais & 2.85 & $1,61-5,04$ & 1.79 & $1,02-3,15$ \\
\hline \multicolumn{5}{|l|}{ Estiveram acamados } \\
\hline Não & 1.00 & & 1.00 & \\
\hline Sim & 1.10 & $0,69-1,73^{*}$ & 1.06 & $0,70-1,58^{\star}$ \\
\hline \multicolumn{5}{|c|}{ Restrição de atividades habituais } \\
\hline Não & 1.00 & & 1.00 & \\
\hline Sim & 1.23 & $0,80-1,88^{*}$ & 0.83 & $0,58-1,20^{*}$ \\
\hline \multicolumn{5}{|c|}{$\begin{array}{l}\text { Renda familiar per capita } \\
\text { em faixas de salário-mínimo }\end{array}$} \\
\hline Mais de 2 SM & 1.00 & & 1.00 & \\
\hline 0,75 a 2 SM & 1.81 & $1,02-3,21$ & 1.09 & $0,78-1,53^{\star}$ \\
\hline Até 0,75 SM & 2.67 & $1,51-4,71$ & 1.18 & $0,84-1,67^{\star}$ \\
\hline Sem renda & 1.65 & $0,77-3,56^{*}$ & 0.98 & $0,60-1,60^{*}$ \\
\hline Sem informação & 0.66 & $0,12-3,53^{*}$ & 1.08 & $0,49-2,36^{*}$ \\
\hline \multicolumn{5}{|l|}{ Plano de Saúde } \\
\hline Não tem & 1.00 & & 1.00 & \\
\hline Um & 1.35 & $0,93-1,96^{*}$ & 1.09 & $0,84-1,42^{*}$ \\
\hline Mais de um & 1.12 & $0,25-4,91^{*}$ & 1.37 & $0,62-3,05^{\star}$ \\
\hline \multicolumn{5}{|c|}{ Serviço de saúde de uso regular } \\
\hline Não & 1.00 & & 1.00 & \\
\hline Sim & 0.90 & $0,66-1,25^{\star}$ & 1.07 & $0,84-1,36^{*}$ \\
\hline
\end{tabular}

* Não significativo.

Salário-mínimo em 1998: R\$130,00.

Nota 1: exclusive a população rural de Rondônia, Acre, Amazonas, Roraima, Pará e Amapá.

Nota 2: exclusive parto normal, cesariana, tratamento psiquiátrico e realização de exames. 
(ou quarta ou mais) internação durante o ano parece configurar um evento independente da primeira internação que parece ser mais prevalente entre os indivíduos de pior condição social, além de estar extremamente associado à precariedade das condições de saúde. Vale destacar que, no caso de internações múltiplas, o efeito da raça não foi observado. Esses resultados reafirmam o sugerido pelos resultados anteriores de que o setor hospitalar tende a responder às demandas das pessoas com pior condição social.

Nunes et al. (2001) estimaram a taxa de internações no SUS em 1999 em 7,19 por 100 habitantes, maior portanto que a taxa de internação geral obtida na análise da PNAD/1998, de 6,9 por 100 habitantes. Essa aparente discrepância é explicada pelo fato de que as informações obtidas do Sistema de Informações Hospitalares do SUS (SIH-SUS) não possibilitam a discriminação de mais de uma internação em um mesmo indivíduo (é uma taxa de internações por população), enquanto que na PNAD/ 1998 a taxa calculada corresponde a pessoas in- ternadas por população, excluindo-se as reinternações, que foram analisadas separadamente.

As médias de permanência hospitalar no Brasil eram superiores à meta prevista pela Consulta Pública Número 1, de 8 de dezembro de 2001, da Secretaria de Assistência à Saúde (SAS) do Ministério da Saúde, que definiu como parâmetros 5,2 dias para clínica médica, 5 dias para pediatria, 4,2 dias para cirurgia, 2 dias para parto normal e 3 dias para parto cirúrgico.

Do ponto de vista da elaboração de políticas públicas, a redução das desigualdades sociais nos aspectos que "capacitam" ao uso de serviços hospitalares reduziriam as desigualdades neste uso. Um sistema que possibilite às pessoas terem "serviço de uso regular", tais como, sistemas com porta de entrada bem definida ou com adscrição das pessoas aos serviços, além de baixo ou nenhum pagamento no ato do consumo, como ocorre paras as pessoas que possuem plano de saúde, seriam medidas de impacto positivo na equidade do consumo de serviços hospitalares no Brasil.

\section{Referências bibliográficas}

Almeida CM, Travassos C, Porto S \& Labra ME 2000. Health Sector Reform in Brazil: a case study of inequity. International Journal of Health Services 30 (1):129-162.

Andersen RM 1968. Behavioral model of families' use of health services. Research Series no. 25. Center for Health Administration Studies, University of Chicago, Chicago.

Andersen RM 1995. Revisiting the behavioral model and access to medical care: does it matter? Journal of Health and Social Behavior 36(1):1-10.

Andersen RM \& Aday LA 1978. Access to medical care in the U.S. realized and potential. Medical Care 16(7): 533-546. 
Anderson GM 1997. Hospital restructuring and the epidemiology of hospital utilization. Medical Care 35(10):OS93-OS91.

Borrell C, Rue M, Pasarín MI, Rohlfs I, Ferrando J \& Fernandez E 2000. Trends in social class inequalities in health status, health-related behaviors, and health services utilization in a Southern European urban area (1983-1994). Preventive Medicine 31:691-701.

Carriere KC, Roos LL \& Dover DC 2000. Across time and space: variations in hospital use during Canadian health reform. Health Services Research 35(2):468487.

Cleary PD \& Jette AM 1984. The validity of self-reported physician utilization measures. Medical Care 22(9): 796-803.

Densen PM, Shapiro S \& Einhorn M 1959. Concerning high and low utilizers of service in a medical care plan, and the persistence utilization levels over a three-year period. The Milbank Memorial Fund Quarterly 37:217-250.

Eriksson I, Unden A \& Elofsson S 2001. Self-rated health: comparisons between three different measures. Results from a population study. International Journal of Epidemiology 30:326-333.

Evans RG 1994. Introduction. In Evans RG, Barer ML \& Marmor TR (orgs.). Why are some people healthy and others not? The determinants of health of populations. Aldine de Gruyter, Nova York.

Freeborn DK, Pope CR, Mullooly JP \& McFarland BH 1990. Consistently high users of medical care among the elderly. Medical Care 28(6):527-540.

Glazier RH, Badley EM, Gilbert JE \& Rothman L 2000. The nature of increased hospital use in poor neighborhoods: findings from a Canadian inner city. Revue Canadienne de Santé Publique 91(4):268-273.

Hofer TP, Wolfe RA, Tedeschi PJ, McMahon LF \& Griffith JR 1998. Use of community versus individual socioeconomic data in predicting variation in hospital use. Health Services Research 33(2):243-259.

Hart JT 1971. The inverse care law. Lancet i:405-412.

Hart JT 2000. Commentary: Three decades of the inverse care law. British Medical Journal 320 (7226):15.

Hulka BS \& Wheat JR 1985. Patterns of utilization: the patient perspective. Medical Care 23(5):438-460.

IBGE 2000. Pesquisa Nacional por Amostra de Domicílios: acesso e utilização de serviços de saúde. Rio de Janeiro.

Jones CP 2001. "Race", racism, and the practice of epidemiology. American Journal of Epidemiology 154(4): 299-304.

Lee ES, Forthofer RN \& Lorimer RJ 1989. Analyzing complex survey data. Sage University Paper Series on Quantitative Applications in the Social Sciences. Sage Pub, Beverly Hills.
McFarland BH, Freeborn DK, Mullooly JP \& Pope CR 1985. Utilization patterns among long-term enrollees in a prepaid group practice health maintenance organization. Medical Care 23:1.221-1.233.

McLaughlin CG, Normolle DP, Wolfe RA, McMahon LF \& Griffith JR 1989. Small-area variation in hospital discharge rates: do socioeconomic variables matter? Medical Care 27(5):507-521.

McMahon JR, Wolfe RA, Griffth JR \& Cuthbertson D 1993. Socioeconomic influence on small area hospital utilization. Medical Care 31:YS29-YS36.

Ministério da Saúde. Consulta Pública número 1, de 8 de dezembro de 2000. Disponível em < http://dtr2001. saude.gov.br/sas/>.

Newbold KB, Eyles J \& Birch S 1995. Equity in health care: methodological contributions to the analysis of hospital utilization within Canada. Social Science and Medicine 40(9):1.181-1.192.

Nunes A, Santos JRS, Barata RB \& Viana SR 2001. Medindo as desigualdades em saúde no Brasil: uma proposta de monitoramento. OPAS/OMS/IPEA, Brasília.

Pinheiro RS \& Travassos C 1999. Estudo da desigualdade na utilização de serviços de saúde por idosos em três regiões da cidade do Rio de Janeiro. Cadernos de Saúde Pública 15(3):487-496.

Porto SM 1997. Eqüidade na distribuição geográfica de recursos em saúde: uma contribuição para o caso brasileiro. Tese de doutoramento, Escola Nacional de Saúde Pública/Fundação Oswaldo Cruz, Rio de Janeiro (mimeo).

Roos NP \& Roos LL 1982. Surgical rate variations: do they reflect the health or socioeconomic characteristics of the population? Medical Care 20:945-958.

Statistical Package for Social Sciences (SPSS) 1997. Release 10.0. SPSS Inc.

Software for the Statistical Analysis of Correlated Data (SUDAAN) 1999. Release 7.5.4. Research Triangle Institute.

Travassos C, Viacava F, Fernandes C \& Almeida CS 2000. Desigualdades geográficas e sociais na utilização de serviços de saúde no Brasil. Ciência e Saúde Coletiva 5(1):133-149.

Travassos C 1997. Eqüidade e o Sistema Único de Saúde: uma contribuição para debate. Cadernos de Saúde Pública 13:325-330.

Travassos C, Sawyer D, Cunha EMGP, Viacava F \& Barro A 2000a. Análise de resultados. In Pesquisa Nacional por Amostra de Domicílios: acesso e utilização de serviços de saúde. IBGE, Rio de Janeiro.

Viacava F, Travassos C \& Sobrinho RP 2000. Relatório sobre gênero. Fiocruz, Rio de Janeiro (mimeo).

Artigo apresentado em 6/3/2002

Aprovado em 12/6/2002

Versão final apresentada em 17/6/2002 